\title{
MBJ-0086 and MBJ-0087, new bicyclic depsipeptides, from Sphaerisporangium sp. 33226
}

\author{
Teppei Kawahara ${ }^{1}$, Masashi Itoh ${ }^{2}$, Miho Izumikawa ${ }^{1}$, Junko Hashimoto ${ }^{1}$, Noriaki Sakata ${ }^{2}$, Toshio Tsuchida ${ }^{2}$ \\ and Kazuo Shin-ya ${ }^{3}$
}

The Journal of Antibiotics (2015) 68, 67-70; doi:10.1038/ja.2014.98; published online 6 August 2014

We have constructed a library of isolated natural products referred to as the 'CB library' for efficient implementation of biological screenings. ${ }^{1}$ To expand and variegate this library, we have conducted chemical and biological screenings using the advanced compoundidentification system based on accumulated HPLC-MS profiling data combined with strain information designated as 'MBJ's special selection'. During screening, we have identified several new natural bioactive compounds such as the cytotoxic eremophilane derivatives MBJ-0009, MBJ-0010, MBJ-0011, MBJ-0012 and MBJ-0013 from Nectria sp. $\mathrm{f} 26111^{2}$ and Apiognomonia sp. $\mathrm{f} 24023,{ }^{3}$ the chaetoglobosin derivatives MBJ-0038, MBJ-0039 and MBJ-0040 from Chaetomium sp. f24230, ${ }^{4}$ a hydroxamate metabolite MBJ-0003 from Micromonospora sp. $29867^{5}$ and the aziridine containing-peptide MBJ-0035 from Streptosporangium sp. $32552 .{ }^{6}$ Further screening was used to isolate new bicyclic depsipeptides designated as MBJ-0086 (1) and MBJ-0087 (2) from the culture broth of Sphaerisporangium sp. 33226 (Figure 1). Here, we describe the fermentation, isolation, structure elucidation and antibacterial activity of $\mathbf{1}$ and 2.

Sphaerisporangium sp. 33226 was isolated from a soil sample collected in Kochi Prefecture, Japan. This producing strain was cultivated in 250-ml Erlenmeyer flasks, each containing $25 \mathrm{ml}$ of a seed medium consisting of $2 \%$ potato starch (Tobu Tokachi Nosan Kako Agricultural Cooperative Assoc., Hokkaido, Japan), 2\% glucose (Junsei Chemical, Tokyo, Japan), 2\% soy bean powder (SoyPro, J-Oil Mills, Tokyo, Japan), 0.5\% yeast extract powder (Oriental Yeast, Tokyo, Japan), $0.25 \% \mathrm{NaCl}$ (Junsei Chemical), $0.32 \% \mathrm{CaCO}_{3}$ (Wako Pure Chemical Industries, Osaka, Japan), 0.0005\% $\mathrm{CuSO}_{4} \cdot 5 \mathrm{H}_{2} \mathrm{O}$ (Wako Pure Chemical Industries), $0.0005 \% \mathrm{ZnSO}_{4} \cdot 7 \mathrm{H}_{2} \mathrm{O}$ (Wako Pure Chemical Industries) and $0.0005 \% \mathrm{MnCl}_{2} \cdot 4 \mathrm{H}_{2} \mathrm{O}$ (Junsei Chemical) for 3 days at $28^{\circ} \mathrm{C}$ on a rotary shaker at 220 r.p.m. $(\mathrm{pH} 7.4)$. The seed culture $(0.5 \mathrm{ml})$ was transferred into $500-\mathrm{ml}$ Erlenmeyer flasks containing $50 \mathrm{ml}$ of the same medium and cultivated for 4 days at $28{ }^{\circ} \mathrm{C}$ on a rotary shaker at 220 r.p.m. After cultivation, an equal volume of $n-\mathrm{BuOH}$ was added to the culture broth (2liters). The $n-\mathrm{BuOH}$ extract was evaporated in vacuo and partitioned between water $(300 \mathrm{ml})$ and EtOAc (300 ml, 3 times).

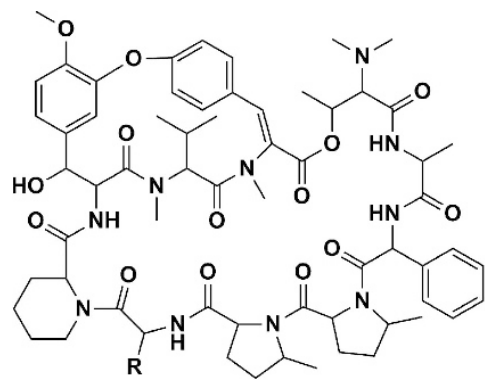

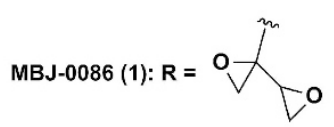<smiles>CC(C)(C)[18O]C#[R]OC1([14CH3])CO1</smiles>

Figure 1 Structures of MBJ-0086 (1) and MBJ-0087 (2).

The EtOAc-soluble material $(1.26 \mathrm{~g})$ was subjected to silica gel medium-pressure liquid chromatography (Si-MPLC, Purif-Pack SI-30, Shoko Scientific, Yokohama, Japan) eluted with a gradient system of $n$-hexane-EtOAc (0-25\% EtOAc) followed by a stepwise solvent system of $\mathrm{CHCl}_{3}-\mathrm{MeOH}(0,2,5,10,20,30$ and $100 \%$ $\mathrm{MeOH})$. Fractions were monitored using the UPLC-DAD-ELS-MS system. The $5 \% \mathrm{MeOH}$ eluate $(553.9 \mathrm{mg}$ ) was subjected to Sephadex LH-20 column chromatography $\left(\mathrm{CHCl}_{3} / \mathrm{MeOH}=1: 1\right.$, GE Healthcare BioSciences AB, Uppsala, Sweden). The target fraction (415.1 mg) was then separated by Si-MPLC (Purif-Pack SI-30) using isocratic elution with $4 \% \mathrm{MeOH}$ in $\mathrm{CHCl}_{3}$ to give fractions $\mathrm{A}(199.5 \mathrm{mg})$ and B $(129.6 \mathrm{mg})$. Fraction A was subjected to a second round of chromatography using Si-MPLC (Purif-Pack SI-30, 3\% $\mathrm{MeOH}$ in $\mathrm{CHCl}_{3}$, isocratically) to obtain crude $2(59.5 \mathrm{mg})$. The pure sample of 2 ( $8.2 \mathrm{mg}$, retention time: $11.0 \mathrm{~min})$ was obtained by two preparative HPLC runs on an XSelect CSH C 18 column $(5.0 \mu \mathrm{m}, 19$ i.d. $\times 150 \mathrm{~mm}$; Waters, Milford, MA, USA) with $55 \%$ aq. $\mathrm{MeCN}$ containing $0.1 \%$ formic acid (flow rate: $10 \mathrm{ml} \mathrm{min}^{-1}$ ). Fraction B was re-chromatographed using Si-MPLC (Purif-Pack SI-30, isocratic, $3 \% \mathrm{MeOH}$ in $\mathrm{CHCl}_{3}$ ) to give semi-purified 1 (51.5 mg). Final purification of 1 was carried out by reversed-phase HPLC (column: CAPCELL PAK C18 MGII column, $5.0 \mu \mathrm{m}, 20$ i.d. $\times 150 \mathrm{~mm}$, Shiseido, Tokyo, Japan; solvent: $40 \%$ aq. MeCN containing $0.1 \%$

1Japan Biological Informatics Consortium (JBIC), Tokyo, Japan; ${ }^{2}$ Bioresource Laboratories, MicroBiopharm Japan Co., Ltd. (MBJ), Shizuoka, Japan and ${ }^{3}$ National Institute of Advanced Industrial Science and Technology (AIST), Tokyo, Japan

Correspondence: Dr K Shin-ya, National Institute of Advanced Industrial Science and Technology (AIST), 2-4-7 Aomi, Koto-ku, Tokyo 135-0064, Japan.

E-mail: k-shinya@aist.go.jp

Received 17 January 2014; revised 8 May 2014; accepted 16 June 2014; published online 6 August 2014 
Table $1{ }^{13} \mathrm{C}(150 \mathrm{MHz})$ and ${ }^{1} \mathrm{H}(600 \mathrm{MHz})$ NMR spectroscopic data for MBJ-0086 (1) and MBJ-0087 (2) in $\mathrm{CDCl}_{3}$

\begin{tabular}{|c|c|c|c|c|}
\hline \multirow[b]{2}{*}{ Position } & \multicolumn{2}{|r|}{1} & \multicolumn{2}{|r|}{2} \\
\hline & $\delta_{C}$ & $\delta_{H}$, mult $(\mathrm{J}$ in $\mathrm{Hz})$ & $\delta_{C}$ & $\delta_{H}$, mult $(\mathrm{J}$ in $\mathrm{Hz})$ \\
\hline
\end{tabular}

\begin{tabular}{|c|c|c|c|c|}
\hline \multicolumn{5}{|c|}{$5 \mathrm{MePro}-1$} \\
\hline 1 & 171.9 & & 172.0 & \\
\hline 2 & 61.1 & $4.38, \mathrm{~d}(7.2)$ & 61.2 & $4.34, d(7.8)$ \\
\hline 3 & 28.8 & $\begin{array}{c}2.63, \text { dd }(7.2,12.6) \\
1.77, \text { ovl }^{a}\end{array}$ & 29.3 & 2.47, m; $1.88, \mathrm{ovl}^{\mathrm{a}}$ \\
\hline 4 & 31.2 & $2.16, \mathrm{ovl}^{\mathrm{a}} ; 1.47, \mathrm{ovl}^{\mathrm{a}}$ & 31.2 & $2.15, \mathrm{ovl}^{\mathrm{a}} ; 1.56, \mathrm{ovl}^{\mathrm{a}}$ \\
\hline 5 & 54.8 & $4.16, \mathrm{~m}$ & 55.1 & 4.17, ovla \\
\hline 6 & 20.8 & $1.35, \mathrm{~d}(6.0)$ & 20.5 & $1.41, \mathrm{~d}(6.0)$ \\
\hline
\end{tabular}

5MePro-2

1.170 .1

$2 \quad 60.6$

$3 \quad 25.9$

$4 \quad 33.1$

$5 \quad 54.6$

$6 \quad 19.71$

PhGly

$\mathrm{NH}$

$1 \quad 167.8^{\mathrm{b}}$

$2 \quad 54.4$

$3 \quad 137.4$

$4 / 8 \quad 128.1$

$5 / 7 \quad 128.4$

$8 \quad 128.0$

Ala

$\mathrm{NH}$

$1 \quad 170.6$

$2 \quad 48.3$

$3 \quad 19.74$

Me2Thr

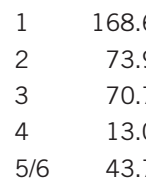

4-MeTyr

$1 \quad 163.2$

$2 \quad 129.5$

$3 \quad 140.3$

$4 \quad 129.6$

$5 \quad 127.5$

$6 \quad 124.9$

$7 \quad 156.6$

$8 \quad 123.1$

$9 \quad 135.2$

$10 \quad 36.0$

MeVal

$1 \quad 170.2$

$2 \quad 56.3$

$3 \quad 29.4$

$4 \quad 19.0$

$5 \quad 18.8$

$\begin{array}{ll}6 & 33.7\end{array}$

$\begin{array}{cr} & 169.8 \\ \text { 4.66, ovl } & 60.5 \\ 2.14, \mathrm{ovl}^{\mathrm{a}} ; 1.96, \mathrm{~m} & 26.0 \\ 2.10, \mathrm{ovl}^{\mathrm{a}}, 1.73, \mathrm{ovl}^{\mathrm{a}} & 33.0 \\ \text { 4.27, m } & 54.8 \\ 0.92, \mathrm{~d}(6.6) & 19.5\end{array}$

7.86, d (7.2)

5.75, d (7.2)

7.45, d (7.8)

7.31, ovl ${ }^{\mathrm{a}}$

7.26, t (7.8)

7.05, ovla

4.58, m

$1.38, \mathrm{~d}(6.6)$

170.4

$48.6^{\mathrm{c}}$

20.0

$\begin{array}{cr}\text { h } & 169.0 \\ 2.95, \text { d }(6.0) & 73.9 \\ 5.08, \text { dq }(6.0,6.6) & 70.6 \\ 1.44, \text { d (6.6) } & 13.1 \\ 2.18, \mathrm{~s} & 43.8\end{array}$

$\begin{array}{cr} & 163.0 \\ & 129.8 \\ & 140.0 \\ & 129.5 \\ & 127.6 \\ 7.32, \text { ovl }^{a} & 124.8 \\ 7.11, \text { dd }(2.4,8.4) & 156.6 \\ & 123.7 \\ 7.13, \text { dd }(2.4,8.4) & 134.7 \\ 7.60, \text { dd }(1.8,8.4) & 36.1 \\ \text { 3.32, s } & \end{array}$

170.1

5.22, d (7.2)

$1.88, \mathrm{~m}$

0.89 , d (7.2)

0.64 , d (7.2)

3.33, s
4.60, dd $(7.8,7.8)$

2.19, ovl ${ }^{\mathrm{a}} ; 1.94, \mathrm{~m}$

2.10, ovla ${ }^{\mathrm{a}}$ 1.71, ovl ${ }^{\mathrm{a}}$

$4.29, \mathrm{~m}$

0.86 , d (6.0)

7.72, d (6.6)

5.82, d (7.2)

7.47, d (7.8)

7.35, dd $(7.8,7.8)$

7.29, ovl ${ }^{\mathrm{a}}$

7.25, ovl ${ }^{\mathrm{a}}$

4.54, m

1.51, d (6.6)

$2.95, \mathrm{~d}(6.0)$

5.17, ovla

1.45, d (6.0)

$2.21, \mathrm{~s}$

$8.10, \mathrm{~s}$

7.30, ovla

7.11 , dd $(1.8,8.4)$

7.19 , dd $(1.8,8.4)$

7.58, d (8.4)

$3.29, \mathrm{~s}$

5.12, d (6.6)

$1.88, \mathrm{ovl}^{\mathrm{a}}$

$0.91, d(6.6)$

$0.67, d(6.6)$

$3.34, \mathrm{~s}$
Table 1 (Continued)

\begin{tabular}{|c|c|c|c|c|}
\hline \multirow[b]{2}{*}{ Position } & \multicolumn{2}{|r|}{1} & \multicolumn{2}{|r|}{2} \\
\hline & $\delta_{C}$ & $\delta_{H}$, mult $(\mathrm{J}$ in $\mathrm{Hz})$ & $\delta_{C}$ & $\delta_{H}$, mult $(J$ in $\mathrm{Hz})$ \\
\hline \multicolumn{5}{|l|}{ mDOPS } \\
\hline $\mathrm{NH}$ & & 7.03, ovla & & 6.95 , br s \\
\hline 1 & 168.9 & & 169.1 & \\
\hline 2 & 59.5 & 4.68, ovla & 59.6 & $4.72, \mathrm{~d}(4.2)$ \\
\hline 3 & 73.86 & 4.95, br s & 74.1 & $4.94, d(10.2)$ \\
\hline 4 & 131.9 & & 131.7 & \\
\hline 5 & 112.0 & 5.32 , br s & 112.2 & 5.28 , br s \\
\hline 6 & 147.1 & & 149.4 & \\
\hline 7 & 149.3 & & 147.2 & \\
\hline 8 & 111.3 & $6.85, \mathrm{~d}(8.4)$ & 111.2 & $6.86, \mathrm{~d}(7.8)$ \\
\hline 9 & 118.7 & $7.06, \mathrm{ovl}^{\mathrm{a}}$ & 118.6 & $7.04, \mathrm{~d}(7.8)$ \\
\hline 10 & 56.0 & $3.92, \mathrm{~s}$ & 56.0 & $3.92, \mathrm{~s}$ \\
\hline $\mathrm{OH}$ & & 6.25 , br s & & $6.29, \mathrm{~d}(10.2)$ \\
\hline \multicolumn{5}{|l|}{ Pip } \\
\hline 1 & 171.1 & & 171.0 & \\
\hline 2 & 54.3 & $5.12, \mathrm{~m}$ & 54.3 & $5.18, \mathrm{ovl}^{\mathrm{a}}$ \\
\hline 3 & 25.5 & 2.22, ovl $^{\mathrm{a}} ; 1.44, \mathrm{ovl}^{\mathrm{a}}$ & 25.6 & 2.27, ovla $; 1.44, \mathrm{ovl}^{\mathrm{a}}$ \\
\hline 4 & 20.2 & $1.59, \mathrm{ovl}^{\mathrm{a}} ; 1.20, \mathrm{~m}$ & 20.2 & $1.61, \mathrm{ovl}^{\mathrm{a}} ; 1.21, \mathrm{ovl}^{\mathrm{a}}$ \\
\hline 5 & 24.6 & $1.70, \mathrm{ovl}^{\mathrm{a}} ; 1.12, \mathrm{~m}$ & 25.0 & 1.59, ovl $^{a} ; 1.19$, ovl $^{\mathrm{a}}$ \\
\hline 6 & 43.2 & $\begin{array}{c}\text { 3.37, br d (14.4); 1.62, } \\
\mathrm{ovl}^{\mathrm{a}}\end{array}$ & 43.8 & $3.66, \mathrm{~m} ; 1.75, \mathrm{ovl}^{\mathrm{a}}$ \\
\hline
\end{tabular}

$\mathrm{Bx}(1) / \mathrm{CeO}(2)$

\begin{tabular}{|c|c|c|c|c|}
\hline $\mathrm{NH}$ & & $9.59, \mathrm{~d}(5.4)$ & & 8.70 , br s \\
\hline 1 & $167.8^{b}$ & & 168.0 & \\
\hline 2 & 51.9 & $4.77, \mathrm{~d}(5.4)$ & 53.2 & $4.88, \mathrm{~d}(6.0)$ \\
\hline 3 & 57.6 & & 58.7 & \\
\hline 4 & 49.5 & $\begin{array}{c}3.08, d(4.2) ; 2.75, d \\
(4.2)\end{array}$ & $48.6^{c}$ & 3.26 , d (3.0); 3.02, br s \\
\hline 5 & 51.4 & $3.48, \mathrm{dd}(2.0,4.0)$ & 69.0 & $4.20, \mathrm{ovl}^{\mathrm{a}}$ \\
\hline 6 & 45.5 & $\begin{array}{l}2.85, \text { dd }(4.0,4.0) \\
2.83, \text { dd }(4.0,4.0)\end{array}$ & 46.4 & $\begin{array}{c}3.84 \text {, dd }(2.4,12.0) ; 3.60 \\
\text { dd }(6.6,12.0)\end{array}$ \\
\hline
\end{tabular}

NMR spectra were taken on a Varian NMR System $600 \mathrm{NB} \mathrm{CL}$ in $\mathrm{CDCl}_{3}$ with the residual solvent peak as an internal standard $\left(\delta_{\mathrm{C}} 77.0, \delta_{\mathrm{H}} 7.25\right.$ p.p.m.).

a Overlapped with other signals.

bInterchangeable.

'Interchangeable.

formic acid; flow rate: $10 \mathrm{ml} \mathrm{min}{ }^{-1}$; retention time: $5.5 \mathrm{~min}$ ) to yield 1 (21.6 mg).

Compound 1 was a colorless amorphous powder; $[\alpha]^{26}{ }_{D}+107$ $(\mathrm{MeOH} ; c$ 0.05). The IR spectrum of 1 showed absorption bands for hydroxy $\left(3400 \mathrm{~cm}^{-1}\right)$ and amide $\left(1640 \mathrm{~cm}^{-1}\right)$ functionalities. The UV spectrum showed absorption maxima at 286 (loge 4.0) and 303 (loge 4.0) nm in $\mathrm{MeOH}$. The molecular formula of 1 was found to be $\mathrm{C}_{67} \mathrm{H}_{86} \mathrm{~N}_{10} \mathrm{O}_{16}$ by high-resolution-ESI-MS $(\mathrm{m} / z 1287.6339$ $[\mathrm{M}+\mathrm{H}]^{+}$, calcd for $\mathrm{C}_{67} \mathrm{H}_{87} \mathrm{~N}_{10} \mathrm{O}_{16}$ 1287.6302). The ${ }^{1} \mathrm{H}$ and ${ }^{13} \mathrm{C}$ NMR spectra of 1 suggested the peptidic nature of this molecule, including 4 amide resonances, signals for $9 \alpha$-methine protons, $4 \mathrm{~N}$-methyl groups and 10 amide carbonyl carbons. The direct connectivity between protons and carbons was determined based on the heteronuclear single quantum coherence spectrum; the tabulated ${ }^{13} \mathrm{C}$ and ${ }^{1} \mathrm{H}$ NMR spectroscopic data for $\mathbf{1}$ are shown in Table 1.

Detailed analyses of DQF-COSY and constant-time $\mathrm{HMBC}^{7}$ spectra revealed the presence of $\mathrm{Ala}, \mathrm{N}-\mathrm{MeVal}$ (MeVal), two 5-MePro (5MePro-1 and 5MePro-2), N,N-diMeThr (Me2Thr), dehydro- $N$ MeTyr ( $\Delta$-MeTyr), $\beta$-hydroxy-4-methoxyTyr (4-methoxydroxidopa, 
a

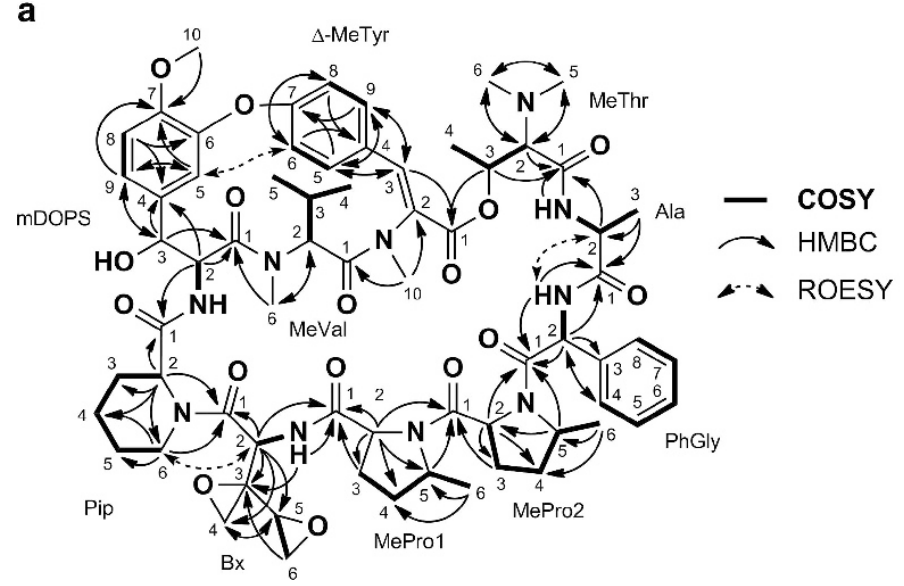

b

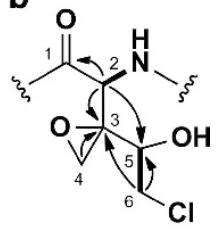

Figure 2 (a) Structure determination of 1. (b) Structure determination of Ceo moiety of 2.

mDOPS), 2-phenylglycine (PhGly) and a pipecolic acid (Pip) residues, as shown in Figure 2a.

The structure of the residual amino acid moiety with a 2,2'-bioxirane moiety (abbreviated as Bx), which is considered to be derived from Ile, was determined based on the COSY correlations from an amide proton $\mathrm{NH}-\mathrm{Bx}\left(\delta_{\mathrm{H}} 9.59\right)$ to a doublet $\alpha$-methine proton $\mathrm{H}-\mathrm{Bx}-2\left(\delta_{\mathrm{H}} 4.77\right)$ and from an oxymethine proton $\mathrm{H}-\mathrm{Bx}-5$ $\left(\delta_{\mathrm{H}} 3.48\right)$ to oxymethylene protons $\mathrm{H}_{2}-\mathrm{Bx}-6\left(\delta_{\mathrm{H}} 2.85\right.$ and 2.83$)$, and $\mathrm{HMBC}$ from the $\mathrm{H}-\mathrm{Bx}-2$ to an amide carbonyl carbon $\mathrm{C}-\mathrm{Bx}-1$ $\left(\delta_{\mathrm{C}} 167.8\right)$, an oxygenated quaternary carbon C-Bx-3 $\left(\delta_{\mathrm{C}} 57.6\right)$, an oxymethylene carbon $\mathrm{C}-\mathrm{Bx}-4\left(\delta_{\mathrm{C}} 49.5\right)$ and an oxymethine carbon $\mathrm{C}-\mathrm{Bx}-5\left(\delta_{\mathrm{C}} 51.4\right)$. High-field shifted ${ }^{13} \mathrm{C}$ NMR chemical values of $\mathrm{C}-\mathrm{Bx}-3, \mathrm{C}-\mathrm{Bx}-4, \mathrm{C}-\mathrm{Bx}-5$ and $\mathrm{C}-\mathrm{Bx}-6\left(\delta_{\mathrm{C}} 45.5\right)$, and the large ${ }^{1} J_{\mathrm{CH}}$ values of $\mathrm{C}-\mathrm{Bx}-4(174 \mathrm{~Hz})$ and $\mathrm{C}-\mathrm{Bx}-6(180 \mathrm{~Hz})$ also supported this bis-epoxide substructure.

The amino acid sequence of 1 was determined based on ${ }^{1} \mathrm{H}-{ }^{13} \mathrm{C}$ long-range couplings from an $\alpha$-proton $\mathrm{H}$-5MePro- $1-2\left(\delta_{\mathrm{H}} 4.38\right)$ to an amide carbonyl carbon C-5MePro-2-1 ( $\left.\delta_{\mathrm{C}} 170.1\right)$, from an $\alpha$-proton H-5MePro-2-2 $\left(\delta_{\mathrm{H}} 4.66\right)$ to an amide carbonyl carbon C-PhGly-1 $\left(\delta_{\mathrm{C}} 167.8\right)$, from an $\alpha$-proton H-PhGly-2 $\left(\delta_{\mathrm{H}} 5.75\right)$ to an amide carbonyl carbon C-Ala- $1\left(\delta_{\mathrm{C}} 170.6\right)$, from an $\alpha$-proton $\mathrm{H}$-Ala$2\left(\delta_{\mathrm{H}} 4.58\right)$ to an amide carbonyl carbon C-Me2Thr-1 $\left(\delta_{\mathrm{C}} 168.6\right)$, from a $\beta$-proton $\mathrm{H}$-Me2Thr-3 $\left(\delta_{\mathrm{H}} 5.08\right)$ to an amide carbonyl carbon of $\Delta$-MeTyr $\left(\delta_{\mathrm{C}} 163.2\right)$, from an $N$-methyl proton of $\Delta$-MeTyr $\left(\delta_{\mathrm{H}} 3.32\right)$ to an amide carbonyl carbon C-MeVal-1 $\left(\delta_{\mathrm{C}} 170.2\right)$, from an $N$-methyl proton of $\mathrm{MeVal}\left(\delta_{\mathrm{H}} 3.33\right)$ to an amide carbonyl carbon C-mDOPS- $1\left(\delta_{\mathrm{C}} 168.9\right)$, from an $\alpha$-proton H-mDOPS-2 $\left(\delta_{\mathrm{H}} 4.68\right)$ to an amide carbonyl carbon C-Pip-1 $\left(\delta_{\mathrm{C}} 171.1\right)$ and from an $\alpha$-proton H-Pip-2 $\left(\delta_{\mathrm{H}} 5.12\right)$ to an amide carbonyl carbon C-Bx-1. The presence of an ether bond between C- $\Delta-$ MeTyr-7 and C-mDOPS- 6 was determined based on the rotating frame NOE between the H- $\Delta$-MeTyr-6 $\left(\delta_{\mathrm{H}} 7.11\right)$ and H-mDOPS-5 $\left(\delta_{\mathrm{H}} 5.32\right)$, and index of hydrogen deficiency deduced from the molecular formula. Furthermore, the ${ }^{1} \mathrm{H}$ and ${ }^{13} \mathrm{C}$ NMR chemical shifts of mDOPS moiety in $\mathbf{1}$ were well matched with those of RA-IV, a cyclic hexapeptide obtained from Rubiae Radix. ${ }^{8}$ Hence, the structure determination of 1 was accomplished as shown in Figure 1. The stereochemistry of the trisubstituted double bond was determined as $Z$ by means of a $J$-resolved HMBC-2 spectrum. ${ }^{9}$ The ester smaller ${ }^{1} \mathrm{H}-{ }^{13} \mathrm{C}$ long-range coupling value of $4.2 \mathrm{~Hz}$ between carbonyl carbon signal of $\Delta$-MeTyr and $\mathrm{H}-\Delta$-MeTyr- 3 indicated that these two nuclei were cis to each other, thus concluding the $Z$ geometry for the double bond. ${ }^{10,11}$
MBJ-0087 (2) was a colorless amorphous powder with the following properties: $[\alpha]^{26}{ }_{\mathrm{D}}+113(\mathrm{MeOH} ; \boldsymbol{c} 0.1)$; UV $\lambda_{\max }(\log \varepsilon)$ in MeOH: 286 (4.0) and 303 (4.0) nm; high-resolution-ESI-MS: $m / z$ $1321.5912[\mathrm{M}-\mathrm{H}]^{-}$, calcd for $\mathrm{C}_{67} \mathrm{H}_{86}{ }^{35} \mathrm{ClN}_{10} \mathrm{O}_{16} \mathrm{~m} / z$ 1321.5912; and IR absorption $\left(v_{\max }\right) 3400$ and $1640 \mathrm{~cm}^{-1}$. Analyses of $1 \mathrm{D}$ and $2 \mathrm{D}$ NMR spectra of $\mathbf{2}$ revealed that the partial structure of $\mathbf{2}$ was the same as that of 1 with the exception of the 2,2'-bioxirane moiety, as described below.

The presence of a 2-amino-2-(2-(2-chloro-1-hydroxyethyl)oxiran2-yl)acetic acid (Ceo) moiety was determined based on COSY correlations from an amide proton $\mathrm{NH}-\mathrm{CeO}\left(\delta_{\mathrm{H}}\right.$ 8.70) to an $\alpha$-methine proton $\mathrm{H}$-Ceo-2 $\left(\delta_{\mathrm{H}} 4.88\right)$, from an oxymethine proton $\mathrm{H}-\mathrm{Ceo}-5\left(\delta_{\mathrm{H}} 4.20\right)$ to chlorinated methylene protons $\mathrm{H}_{2}$-Ceo-6 $\left(\delta_{\mathrm{H}} 3.84,3.60\right)$, and ${ }^{1} \mathrm{H}-{ }^{13} \mathrm{C}$ long-range couplings from the $\mathrm{H}-\mathrm{Coe}-2$ to an amide carbonyl carbon C-Coe-1 $\left(\delta_{\mathrm{C}} 168.0\right)$, an oxygenated quaternary carbon C-Coe-3 $\left(\delta_{\mathrm{C}} 58.7\right)$ and an oxymethine carbon C-Coe-5 $\left(\delta_{\mathrm{C}} 69.0\right)$, from oxymethylene protons $\mathrm{H}_{2}$-Ceo-4 $\left(\delta_{\mathrm{H}} 3.26,3.02\right)$ to the C-Coe-3 and from the $\mathrm{H}_{2}-\mathrm{Ceo}-6$ to the C-Coe-3 and C-Coe-5, and low-field shifted chemical shifts of $\mathrm{C}-\mathrm{Ceo}-3$ and C-Ceo-4 $\left(\delta_{\mathrm{C}} 48.6\right.$; Figure $\left.2 \mathrm{~b}\right)$. HMBCs from the $\mathrm{H}-\mathrm{Ceo}-2$ to an amide carbonyl carbon C-5MePro-1-1 $\left(\delta_{\mathrm{C}} 172.0\right)$ and from an $\alpha$-methine proton $\mathrm{H}$-Pip-2 $\left(\delta_{\mathrm{H}} 5.18\right)$ to $\mathrm{C}-\mathrm{Ceo}-1$ revealed the gross structure of 2 as shown in Figure 1.

Antimicrobial activity against Bacillus subtilis was tested. First, $20 \mu \mathrm{l}$ of a log-phase B. subtilis culture $\left(2 \times 10^{6} \mathrm{CFU} \mathrm{ml}^{-1}\right)$ was dispensed into each well in a 384-well plate and compounds were added at various concentrations. Dimethyl sulfoxide (1\%) was used as a vehicle control. After incubation for $18 \mathrm{~h}$ at $37^{\circ} \mathrm{C}$, the $\mathrm{OD}_{620}$ was measured using a 2103 Envision Multilabel Reader (PerkinElmer, Waltham, MA, USA). Compounds 1 and 2 were active against $B$. subtilis with $\mathrm{IC}_{50}$ values of 1.1 and $24 \mu \mathrm{M}$, respectively. In contrast, neither compound showed cytotoxicity against human ovarian adenocarcinoma SKOV-3 cells $\left(\mathrm{IC}_{50}>50 \mu \mathrm{M}\right)$ or antimicrobial activity against Micrococcus luteus $\left(\mathrm{IC}_{50}>50 \mu \mathrm{M}\right)$.

The structures of $\mathbf{1}$ and $\mathbf{2}$ are extremely unique and include bicyclic depsipeptides, diphenyl ether and rare amino acid residues such as 5-MePro, $\Delta$-MeTyr, mDOPS and Bx (1) or Ceo (2). Compounds 1 and 2 are the first natural peptide compounds possessing $\mathrm{Bx}(\mathbf{1})$ or a Ceo (2) units, respectively. Furthermore, there have been no reports describing peptide compounds possessing a diphenyl ether moiety except for dityromycin ${ }^{12}$ isolated from Streptomyces sp. and the bicyclic hexapeptides isolated from Rubiaceae. ${ }^{13,14}$ 


\section{ACKNOWLEDGEMENTS}

This work was supported in part by a grant from the New Energy and Industrial Technology Department Organization (NEDO) of Japan, and a Grant-in-Aid for Scientific Research (23380067 to KS-y) from the Japan Society for the Promotion of Science (JSPS).

1 Kawahara, T., Nagai, A., Takagi, M. \& Shin-ya, K. JBIR-137 and JBIR-138, new secondary metabolites from Aspergillus sp. fA75. J. Antibiot. 65, 535-538 (2012).

2 Kawahara, T. et al. Cytotoxic sesquiterpenoids MBJ-0009 and MBJ-0010 from saprobic fungus Nectria sp. f26111. J. Antibiot. 66, 567-569 (2013).

3 Kawahara, T. et al. Three eremophilane derivatives, MBJ-0011, MBJ-0012 and MBJ-0013, from an endophytic fungus Apiognomonia sp. f24023. J. Antibiot. 66 299-302 (2013).

4 Kawahara, T. et al. New chaetoglobosin derivatives, MBJ-0038, MBJ-0039 and MBJ-0040 isolated from the fungus Chaetomium sp. f24230. J. Antibiot. 66, 727-730 (2013).

5 Kawahara, T. et al. New hydroxamate metabolite, MBJ-0003, from Micromonospora sp. 29867. J. Antibiot. 67, 261-263 (2014).
6 Kawahara, T. et al. Novel aziridine-containing peptides MBJ-0034 and MBJ-0035 from Streptosporangium sp. 32552. J. Antibiot. 67, 577-580 (2014).

7 Furihata, K. \& Seto, H. Constant time HMBC (CT-HMBC), a new HMBC technique useful for improving separation of cross peaks. Tetrahedron Lett. 39, 7337-7340 (1998)

8 Itokawa, H. et al. Studies on antitumor cyclic hexapeptides RA obtained from Rubiae Radix, Rubiaceae. VI. Minor antitumor constituents. Chem. Pharm. Bull. 34 3762-3768 (1986).

9 Furihata, K. \& Seto, H. J-Resolved HMBC, a new NMR technique for measuring heteronuclear long-range coupling constants. Tetrahedron Lett. 40, 6271-6275 (1999).

10 Palermo, J. A. et al. Celenamide E, a tripeptide alkaloid from the Patagonian sponge Cliona chilensis. J. Nat. Prod. 61, 488-490 (1998).

11 Shigematsu, N. et al. Structure of WS9326A, a novel tachykinin antagonist from a Streptomyces. J. Org. Chem. 58, 170-175 (1993).

12 Teshima, T. et al. The structure of an antibiotic, dityromycin. Tetrahedron Lett. 29, 1963-1966 (1988)

13 Jolad, S. D. et al. Bouvardin and deoxybouvardin, antitumor cyclic hexapeptides from Bouvardia ternifolia (Rubiaceae). J. Am. Chem. Soc. 99, 8040-8044 (1977).

14 Singh, R., Geetanjali \& Chauhan, S. M. 9,10-Anthraquinones and other biologically active compounds from the genus Rubia. Chem. Biodivers. 1, 1241-1264 (2004).

Supplementary Information accompanies the paper on The Journal of Antibiotics website (http://www.nature.com/ja) 